\title{
Two Degree-of-Freedom Resonant Structure for Robust Current Control and Active Power Filtering
}

\author{
Naim Bajcinca \\ GERMAN AEROSPACE CENTER (DLR) \\ Institute of Robotics und Mechatronics \\ Münchnerstr.20, 82234 Oberpfaffenhofen \\ Tel: 08153-28-2453 \\ Fax: 08153-28-1441 \\ Email: naim.bajcinca@dlr.de
}

\author{
Dietrich Naunin \\ UNIVERSITY OF TECHNOLOGY OF BERLIN \\ Institute of Energy and Automation Technology \\ Einsteinufer 17, 10587 Berlin \\ Tel: 030-314-24207 \\ Fax: 030-314-22120 \\ Email: naunin@tubife1.ee.tu-berlin.de
}

\section{Keywords}

«2 DOF control», «Current control», «Active power filtering»

\begin{abstract}
This paper introduces a novel two degree-of-freedom resonant control structure for current control in a stationary reference frame in one- or three-phase power electronics systems. The main focus is put on the design of the controller for robust tracking of a single or a linear combination of harmonic components, while providing robustness with respect to parameter variations, disturbance and measurement noise. The method is applied for current control of a single phase active power filter.
\end{abstract}

\section{Introduction}

Many applications in power electronics inverter meet the problem of current control within a defined frequency spectrum. A typical class of such applications are active power filters. For instance, a shunt active power filter is connected in parallel with the nonlinear load and operates as a controlled current source for cancellation of the current harmonic and reactive components generated by the load. In the case of three-phase systems a standard control approach uses $d$ - $q$ transformation of harmonic signals with fundamental frequency into dc signals in a rotating reference frame, [4]. Alternatively, solutions for control in stationary coordinates have been also recently proposed, [2]. Given that no $d-q$ transformations are required, the stationary reference approach applies also for single phase systems.

This paper proposes a two degree-of-freedom control structure for control of harmonic inputs in stationary reference frame. The method presented here provides extensions to the basic design discussed in [1] for robust tracking of harmonic references. The controller, designated also as inverse disturbance observer (IDOB), combines the principle of feedforward inversion and high-gain feedback in a very simple structure. In this paper special emphasis is put on the design of the high-gain feedback loop. For robust tracking of a harmonic reference with a frequency $\omega_{0}$ the $Q$ loop is designed to resonate at the same frequency. The key issue here is that this resonance is independent on the plant dynamics and its parameter variations, thus providing perfect robust tracking of harmonic references. Its design is further generalized for simultaneous tracking of a linear combination of a finite set of harmonic components.

The paper is organized in three sections. The control structure and its basic properties are discussed in detail in the first section. In the second section the controller is applied for robust current control of a single phase PWM inverter. The control design for a single phase active power filter is shown in the third section. The controller is validated by simulations in both cases. 


\section{Two degree-of-freedom structure}

\section{A. Basic idea}

The inverse disturbance observer (IDOB) two degree-of-freedom structure, [1] , in its basic form is shown in Fig 1. Identify here the plant $G$, and the two design parameters (two degrees of freedom): $\tilde{G}^{-1}$ the approximate inverse of $G$, and the $Q$-filter.

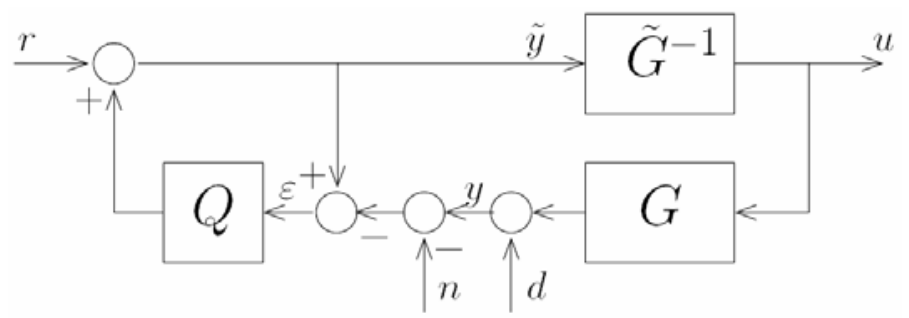

Fig 1: Two degree of freedom controller

The transfer functions from the reference $r$ to the plant input $u\left(G_{r u}\right)$ and its output $y\left(G_{r y}\right)$ are easily obtained from Fig 1

$$
G_{r u}=\frac{\tilde{G}^{-1}}{1-Q\left(1-\tilde{G}^{-1} G\right)} \quad \text { and } \quad G_{r y}=\frac{\tilde{G}^{-1} G}{1-Q\left(1-\tilde{G}^{-1} G\right)}
$$

To present the basic idea of the IDOB control structure introduce the notation:
$(A): Q=1$
(infinity gain feedback)
(B) : $\tilde{G}^{-1}=G^{-1}$
(perfect model inversion)
(C): $G_{r y}=1$
(perfect input tracking).

Then equation (1) reads directly $(A) \vee(B) \Rightarrow(C)$, that is $(C)$ applies if any of $(A)$ and $(B)$ is true. In practice, none of the conditions $(A)$ and $(B)$ is realizable for all frequencies. In fact, $(A)$ would destabilize any practical loop, so $Q$ must ultimately roll-off for high-frequencies. Further, the feedforward exact inversion as defined in $(B)$ fails at least for high-frequencies. Hence, $(A)$ and $(B)$ make sense only in the operational frequency bandwidth. However, even then the two conditions must be weakened due to implementation limitations to
$\left(A^{\prime}\right): Q \approx 1$
(high-gain feedback)
$\left(B^{\prime}\right): \tilde{G}^{-1} \approx G^{-1}$
(approximate model inversion).

It is important that though weakened $\left(A^{\prime}\right)$ and $\left(B^{\prime}\right)$ contribute independently towards perfect tracking $G_{r y}=1$. Therefore, IDOB control structurally unifies the high-gain feedback and the feedforward exact inversion principle.

\section{B. Robustness}

Essential implications for the IDOB structure are provided by the sensitivity $S=y / d$ and complementary sensitivity $S=y / n$ functions 


$$
S=\frac{1-Q}{1-Q\left(1-\widetilde{G}^{-1} G\right)} \text { and } T=\frac{Q \tilde{G}^{-1} G}{1-Q\left(1-\widetilde{G}^{-1} G\right)} .
$$

By definition, $S=1 /(1+L)$ and $T=L /(1+L)$, where $L$ stands for the loop transfer function

$$
L=\frac{Q \tilde{G}^{-1} G}{1-Q} .
$$

It is convenient to define also the three latter functions for the situation that corresponds to feedforward perfect inversion, condition $(B)$

$$
\tilde{S}=1-Q, \quad \tilde{T}=Q \quad \text { and } \quad L=\frac{Q}{1-Q} .
$$

Since $\tilde{G}^{-1}$ provides just an approximate inversion of the plant $G$, it is natural to introduce the notion of imperfect inversion. For the sake of simplicity consider the multiplicative imperfection $G=\tilde{G}^{-1}\left(1+W_{2} \Delta\right)$, where $W_{2}$ is a proper stable weighting function and $|\Delta|_{\infty} \leq 1$ represents a stable unstructured disk like uncertainty. Note that typically $W_{2}$ increases with frequency due to model mismatching. A natural question reads as follows: assuming that IDOB closed loop with perfect inversion is stable, how big is the minimal imperfection that destabilizes the closed loop? In [1] it is shown that robust stability is guaranteed if and only if the condition

$$
\left|W_{2} Q\right|_{\infty}<1
$$

holds. Equation (5) sets stability constraints in the interaction between the imperfection $W_{2}$ and the design parameter $Q$. It has a simple and elegant geometrical interpretation in terms of the Bode plots of $Q$ and $W_{2}$. Namely, the IDOB structure with the imperfection $W_{2}$ is internally stable if and only if the magnitude Bode plot of the function $W_{2}^{-1}$ lies above that of $Q$. Recall that $\left|W_{2}^{-1}\right|$ rolls off toward zero at high frequencies, thus putting a limitation for the bandwidth of the filter $Q$.

\section{Tracking}

Asymptotic tracking is defined as the ability of the control loop to drive $y \rightarrow r$ as $t \rightarrow \infty$. For instance, if $r$ is a step, then due to

$$
\frac{e}{r}=S\left(1-\tilde{G}^{-1} G\right)
$$

where $e=y-r$, the function $S$ must have a zero at $s=0$, which is further equivalent to $Q(0)=1$. For example this is fulfilled by

$$
Q=\frac{1}{\tau \mathrm{s}+1}
$$

Now consider a set of sinusoidal inputs confined within some frequency bandwidth. Let $W_{p}$ weight a desired tracking response in (6) in the sense that $|e|_{2}<\left|W_{p}^{-1}\right|_{\infty}|r|_{2}$. For larger $\left|W_{p}\right|$ the tracking performance improves. However, it is intuitive that for sufficiently large imperfections $\left|W_{2} \Delta\right|$, the performance set by $W_{p}$ may get lost. It is thus important to set up the conditions for meeting the 
performance set by $W_{p}$ in the presence of the imperfections $W_{2}$. Therefore, substitute $G=\tilde{G}^{-1}\left(1+W_{2} \Delta\right)$ into (6) to get $\left|W_{1} S \Delta\right|_{\infty}<1, \forall \omega$, or equivalently

$$
\left|W_{1} S\right|_{\infty}<1
$$

where we switch to the notation $W_{1}=W_{p} W_{2}$. This equation can be further manipulated provided that internal stability condition $\left|W_{2} Q\right|_{\infty}<1$ holds to

$$
|| W_{1}(1-Q)|+| W_{2} Q \|_{\infty}<1 .
$$

Equation (9) is an elegant description of simplicity and efficiency of the IDOB control structure. It represents the basic equation for the design by loopshaping $Q$. It can be shown that thereby a very simple

$Q$ results. Namely, within the operational frequency bandwidth, where $\left|W_{1}\right|>>\left|W_{2}\right|$ holds, the condition $Q \approx 1$ results, and for high frequencies ( $\left.\left|W_{2}\right|>>\left|W_{1}\right|\right), Q \approx 0$ results. In general, it is easy to design an appropriate $Q$. E.g. for the $Q$ in (7) the only design parameter is its bandwidth $1 / \tau$. For its design equation (9) should be used. Note that, for better performance the term $\left|W_{1}(1-Q)\right|$ in (9) requires a high bandwidth, while being compelled by the stability term $\left|W_{2} Q\right|$. Thus, the designer should meet a compromise between these two conflicting specifications.

\section{Harmonic tracking}

Tracking of harmonic references is the key issue in this paper. Therefore we show now how can $Q$ be designed such that perfect tracking holds regardless of imperfection $W_{2}$. Without loss of generality let the reference be a sinusoid $r=\sin \omega_{0} t$. Going back to the performance specification $|e|_{2}<\left|W_{p}^{-1}\right|_{\infty}|r|_{2}$, we need to set $W_{p}\left(j \omega_{0}\right)=\infty$, which due to $W_{1}=W_{p} W_{2}$ is equivalent to $W_{1}\left(j \omega_{0}\right)=\infty$. But according to (9) this is possible if $Q\left(j \omega_{0}\right)=1$. Similarly, if $Q\left(j \omega_{0}\right)=1$, then $W_{p}\left(j \omega_{0}\right)=\infty$ must hold, too. The lowest order $Q$-filter which satisfies this condition is of the form

$$
Q=\frac{2 D \omega_{b} s+\left(\omega_{b}{ }^{2}-\omega_{0}{ }^{2}\right)}{s^{2}+2 D \omega_{b} s+\omega_{b}{ }^{2}}
$$

where $0<D \leq 1$ stands for the damping and $\omega_{b} \geq \omega_{0}$ for the bandwidth of the filter $Q$. Given that the loop transfer function is $L=Q /(1-Q)$, a resonance effect appears at the frequency $\omega=\omega_{0}$, and no matter what imperfection, perfect asymptotic tracking applies.

\section{E. Disturbance and noise suppression}

Up to now we have been considering the reaction of the control loop in Fig 1 only regarding to the input $r$. The focus here is set to the response with respect to the other two inputs: $d$ (disturbance) and $n$ (measurement noise). More precisely the issue is the design of $Q$, such that robust performance (9) is not endangered by these undesirable inputs. Note that according to (2), the latter may be represented in the form $\left|W_{1} \tilde{S}\right|+\mid W_{2} \tilde{T} \|_{\infty}<1$. The previous considerations have shown that within the operational frequency 
bandwidth, $S$ must be kept as low as possible, while $T$ is to be reduced at high frequencies. Fortunately, the effect of $d$ and $n$ is thereby simultaneously suppressed since usually $d$ appears in the operational frequencies, and $n$ at high frequencies. In other words, the situation $T \approx 1$ in the operational range is not critical, since the measurement noise $n$ is therein negligible, and in the noise suppression range $S \approx 1$ is harmless since $d$ is low.

\section{F. Structural offset compensation}

In some situations, the measurement noise may yet be low frequent in the form of an offset. In practice, it is very often the case that sensors are offset. Such a situation is typical when a sensor which measures relative differences is switched on. Alternatively, offset may occur due to exogenous effects, such as temperature. Disturbance differs from offset in that that it resembles a real physical signal, while offset does not. Offset is especially dangerous in systems with integral control as the case with IDOB is. Basically, offset can be modelled by a constant noise. Thus for its suppression $T(0)=0$ is required, or equivalently $Q(0)=0$, see (2). To implement this condition the filter $Q$ is modified by setting $\omega_{b}=\omega_{0}$ in (10)

$$
Q=\frac{2 D \omega_{0} s}{s^{2}+2 D \omega_{0} s+\omega_{0}{ }^{2}}
$$

\section{Robust current control of PWM inverter}

Consider first the current control problem of a single-phase PWM inverter with a R-L circuit load. The task here is tracking of the input $i_{s}$ at the output $i$. The corresponding control scheme using the IDOB control structure is shown in Fig 2. Here $G$ stands for the impedance of the series R-L circuit

$$
G=\frac{1}{R+L s}
$$

and $\widetilde{G}^{-1}=\widetilde{R}+\widetilde{L} s$ for its inverse. Notice that $\widetilde{G}^{-1}$ in this form cannot be realized due to causality constraints. Therefore the filter $Q$ is relocated as shown in Fig 2. Thereby, all essential structural features discussed in the previous section are preserved. The denotation $\sim$ declares that the load parameters may change.

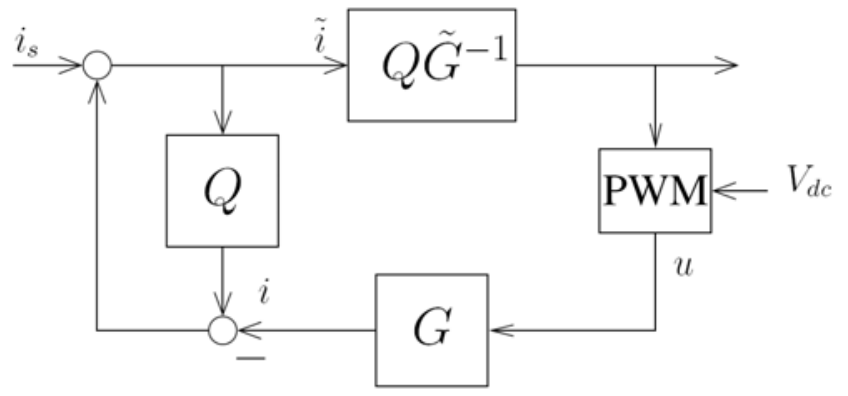

Fig 2: Current control structure 
Assume that the reference current is given by $i_{s}=\sin \omega_{0} t$. Here the two $Q$ filters in (10) and (11) are tested. The simulation results for the set of parameters $\widetilde{R}=10, \widetilde{L}=80 \cdot 10^{-3}, \omega_{0}=2 \pi 50$, and $V_{d c}=100$ in SI units are shown in Fig 3. the carrier frequency of the PWM inverter is $10 \mathrm{kHz}$. The first row shows the current tracking response with the $Q$ filter from (11) and (12), respectively. In the latter case, $\omega_{b}=10 \omega_{0}$ is set. The second row shows the respective tracking error. In both cases at some $t$ the load resistance changes instantaneously from $R=10$ to $R=20$. Notice that the controller compensates the error within less then one period, thus demonstrating robustness of the structure in Fig 2. While due to parameter changes the exact inversion in the feedforward loop is violated, the error is eliminated because of the resonance of the $Q$ loop at the input frequency. It is obvious that the tracking performance with the $Q$ filter from (12) is superior to that from (10). This is simply explained by the fact that for the frequencies around the resonant frequency $\omega_{0}$, the $Q$ loop in (10) with $\omega_{b}>\omega_{0}$ exhibits higher gains. Note that during the transient phase such frequencies are excited by the changes in the load parameter $R$.
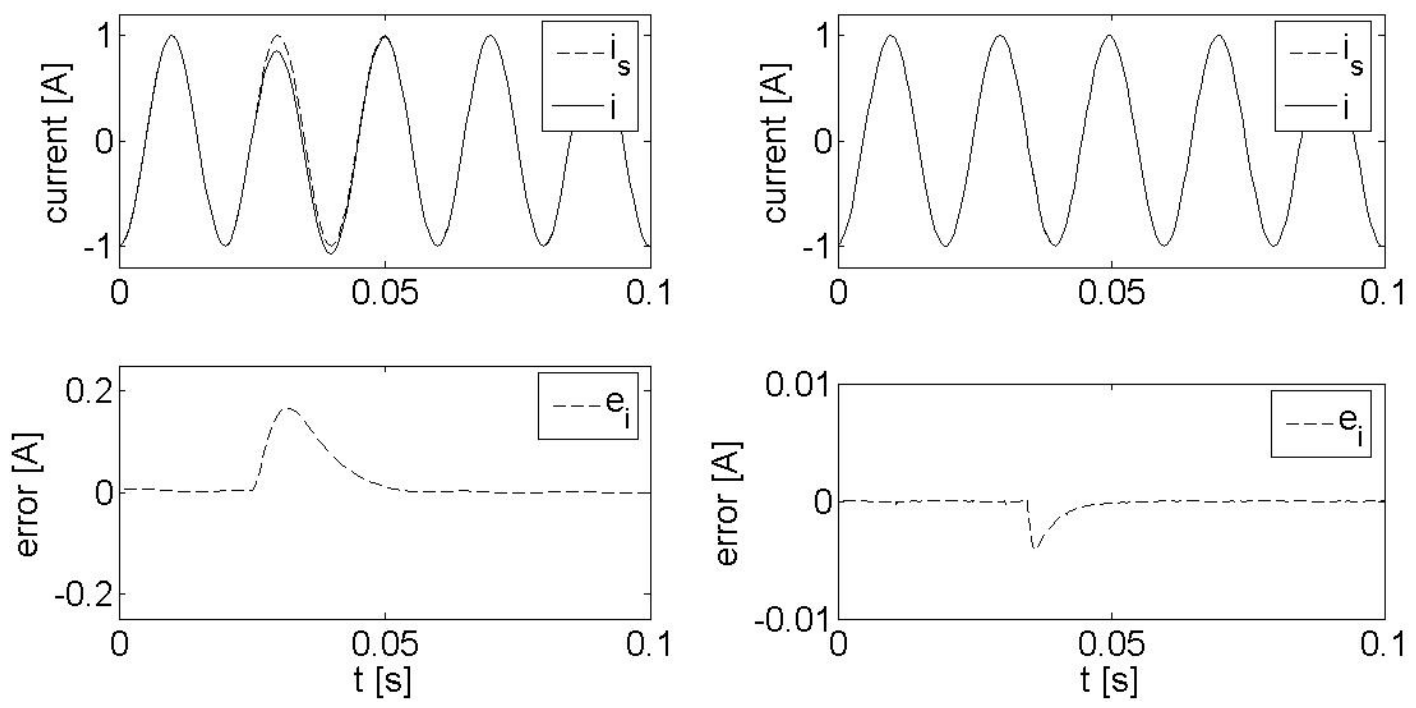

Fig 3: Simulation results with (left) $Q$ given by (10), $\omega_{b}=10 \omega_{0}$, and (right) $Q$ given by (11)

\section{Single phase active power filter}

\section{A. Problem definition}

Fig 4 shows the topology of a single phase shunt active power filter, which operates as a current source for cancellation of harmonics generated by the nonlinear load for improvement of the load power factor. As a result, the power distribution system sees the net load as a pure resistance, so the source current $i_{e}$ contains only the fundamental harmonic. The harmonic components are extracted by sensing the load current $i_{l}$. As a nonlinear load here a diode rectifier and a capacitive load are used. 


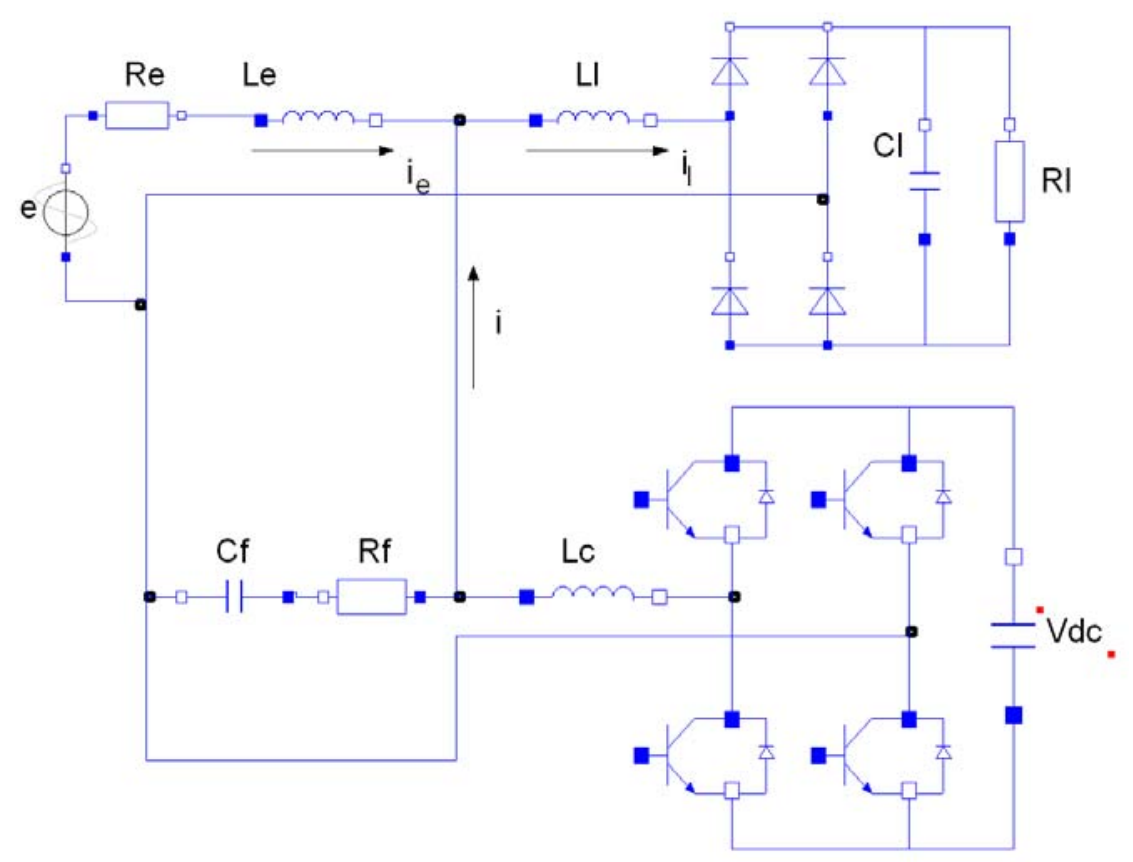

Fig 4: Shunt active power filter

For the system in Fig 4, the load current $i_{l}$ may be represented as the sum of an infinite series of odd harmonic components

$$
i_{l}=\sum_{j=0}^{\infty} I_{j} \sin \left((2 j+1) \omega_{0} t+\varphi_{j}\right)
$$

whereby in the practical sense just first several harmonics are relevant. Therefore the reference signal in this case is of the form

$$
i_{s}=I_{3} \sin \left(3 \omega_{0} t+\varphi_{3}\right)+I_{5} \sin \left(5 \omega_{0} t+\varphi_{5}\right)+\ldots+I_{m} \sin \left(m \omega_{0} t+\varphi_{m}\right)
$$

The task here is to track this reference at the injection current $i$ in the scheme of Fig 4. The basic challenge in this case is generalization of the IDOB control structure for simultaneous perfect tracking of multiple harmonic components. Besides, the generator $e$ acts as a disturbance for the open loop, and the load parameters may change.

By superposition theorem the injection current $i$ of the active filter in Fig 4 can be approximately modelled as

$$
i=G_{i} u+G_{e} e .
$$

whereby $u$ stands for the output of the PWM inverter, and $e$ for the generator of sine voltage. For the computation of $G_{i}$ and $G_{e}$ the diode rectifier block will be approximated by a unity gain. They are obviously fifth order transfer functions. While their exact computation is omitted here, the reader may check that in the operational frequency range $G_{i}$ can be approximated by a second order transfer function. 


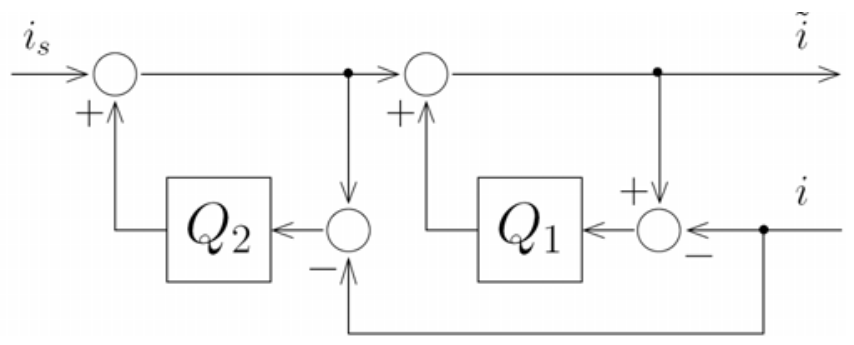

Fig 5: Cascading $Q$ loops

\section{B. Control structure}

The feedforward loop can be designed in two different ways. One can make use of disturbance suppression of the IDOB structure by considering the term $G_{e} e$ as an output disturbance. The control law in this case would be

$$
u=Q \tilde{G}_{i}^{-1} \tilde{i} .
$$

Alternatively, given the signal $e$, one may compensate for its disturbing effect by the following control law

$$
u=Q \tilde{G}_{i}^{-1}\left(\tilde{i}-G_{e} e\right)
$$

The $Q$ filter is here proposed in the form

$$
Q=1-\left(1-Q_{1}\right)\left(1-Q_{2}\right) \ldots\left(1-Q_{m}\right)
$$

where $Q_{j}$ are given by (10) or (11) with a resonance at the corresponding frequency $\omega_{j}$, $j=1,2, \ldots, m$. Note that $Q\left(j \omega_{j}\right)=1$, for any $j$, thus according to (2), $S\left(j \omega_{j}\right)=0$, and referring to (9) for any imperfection (uncertainty) $W_{2}$, the performance weighting $W_{p}\left(j \omega_{j}\right) \rightarrow \infty$. Hence perfect robust tracking holds at any resonant frequency $\omega_{j}$. It is interesting to note that it is a matter of several block algebra steps to show that actually the filter of the form (18) is equivalent to a cascade structure of the $Q$ loops as shown in Fig 5 (for $m=2$ ), which may be important for implementation aspects. The resulting control structure using (17) is shown in Fig 6.

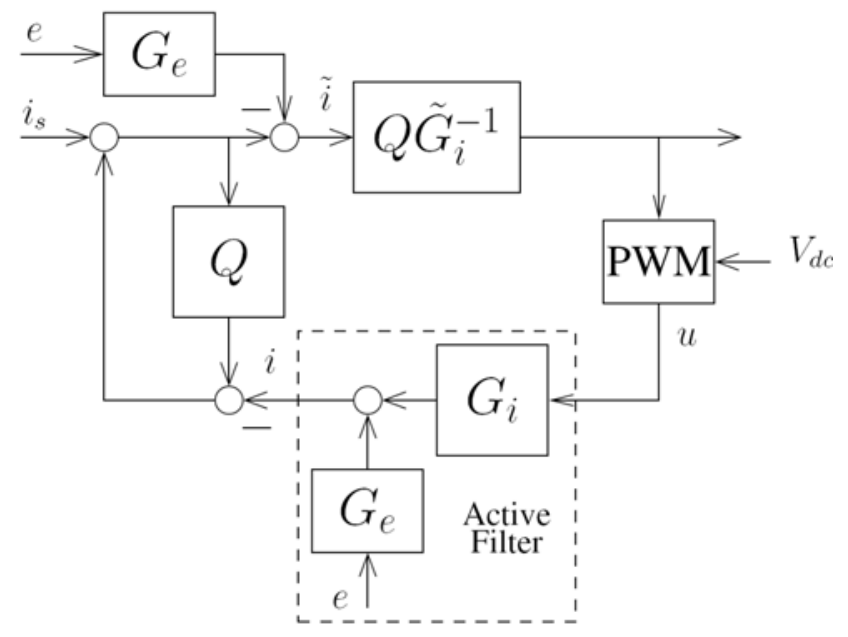

Fig 6: Active power filter control structure 


\section{Simulation results}

The simulation results with the structure in Fig 6 are shown in Fig 7. The first column of plots shows the response of the load $\left(i_{l}\right)$ and source $(i)$ current, and the second one the reference $\left(i_{s}\right)$ and measured $(i)$ injection current. To demonstrate robustness two situations are checked: the first row of plots corresponds to the load resistance $R_{l}=10$, and the second one to $R_{l}=30 \mathrm{ohms}$. In both cases three first harmonics 3, 5 and 7 are cancelled. To show the transient response the controller is switched on after the first period. The filtering effect is clearly demonstrated by the left plots. On the other hand, the right plots demonstrate robust perfect tracking of injection current harmonics. The used parameters in SI units are as follows: $R_{e}=1, L_{e}=10^{-3}$, $L_{l}=5 \cdot 10^{-3}, C_{l}=150 \cdot 10^{-6}, L_{c}=5 \cdot 10^{-3}, R_{f}=5.5, C_{f}=4 \cdot 10^{-6}, \omega_{0}=2 \pi 50$, and $V_{d c}=100$.
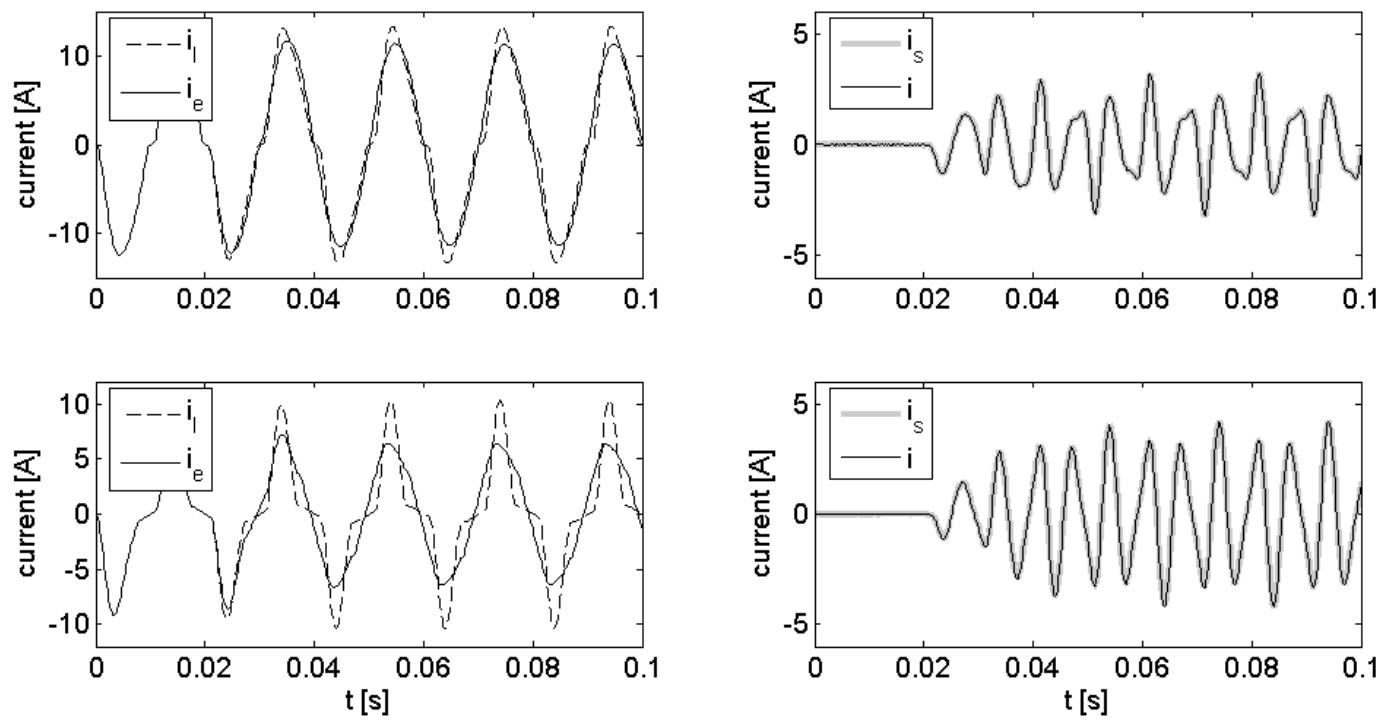

Fig 7: Simulation results for active filtering

\section{Conclusion}

A novel two degree-of-freedom control structure for power electronics inverter applications is introduced for tracking of harmonic references in stationary reference frame. The method is characterized by its design simplicity due to the clear physical description of its design parameters. The designer needs to design an inverse of the plant $\widetilde{G}^{-1}$, and a simple filter $Q$ which at resonance frequencies equals one. Here a second order filter is used therefore. Its resonant frequency is determined by the harmonic reference. If a sum of harmonics is to be tracked, then it is shown that an appropriate structure is realized by cascading the set of $Q$ loops with resonances at corresponding harmonic frequencies. Thereby perfect tracking is guaranteed regardless on possible imperfections in the plant inverse $\tilde{G}^{-1}$. The resulting controller is shown to be effective for the design of active power filters. While their design is illustrated in the single phase case, the controller is directly applicable in three phase systems, as an alternative to $d$ - $q$ transformation. 


\section{References}

[1]. N. Bajcinca, T. Bünte. A novel control structure for inversion and tracking tasks, XVI IFAC World Congress, Prague 2005

[2]. S. Fukuda, T. Yoda. A novel current tracking method for active filters based on a sinusoidal internal model, IEEE Transaction on industry applications, Vol 37, No 3, 2001.

[3]. R.F. Pinheiro, C.B. Jacobina, A.M.N. Lima E.R.C. da Silva: A revision of the state of the art in active filters

[4]. N. Mendalek, K. Al-Haddad: Modeling and nonlinear control of shunt active power filter in the synchronus reference frame, Proc. of $9^{\text {th }}$ International Conference on Harmonics and Quality Power, Vol $1,2000$. 